\title{
Online single-shot pulse reconstruction for optimizing a seeded X-ray free-electron laser
}

\author{
Li Zenga ${ }^{\mathrm{a}, \mathrm{b}}$, Duan $\mathrm{Gu}^{\mathrm{c}}$, Chao Feng ${ }^{\mathrm{c}, *}$, Bo Liu ${ }^{\mathrm{c}, *}$, Zhentang Zhao ${ }^{\mathrm{c}}$ \\ ${ }^{a}$ Shanghai Institute of Applied Physics, Chinese Academy of Sciences, Shanghai 201800, China \\ ${ }^{b}$ University of Chinese Academy of Sciences, Beijing 100049, China \\ ${ }^{c}$ Shanghai Advanced Research Institute, Chinese Academy of Science, Shanghai 116023, China
}

\begin{abstract}
X-ray free-electron lasers (FELs) hold promising prospects for opening up opportunities for ultra-fast sciences at the atomic and molecular system. A precise knowledge of temporal information of FEL pulses is the central issue for experiments. Here we demonstrated an online diagnostic method to determine the FEL temporal profiles at the Shanghai Soft X-ray FEL facility. This robust method, designed for seeded FELs, allows researchers to acquire real-time longitudinal profiles of FEL pulses with a resolution better than 3 fs. Based on this method, for the first time, we can directly observe the generation and evolution of a seeded FEL online. This helps us to further understand the physics and realize the lasing of a stable, nearly fully coherent soft X-ray FEL through a two-stage harmonic up-shift configuration. This method also provides an intuitive way for precise detection and control of the relative timing between electron beams and external optical lasers.
\end{abstract}

\section{Introduction}

\footnotetext{
${ }^{*}$ Corresponding authors

Email addresses: fengchao@zjlab.org.cn (Chao Feng), liubo@zjlab.org.cn (Bo Liu)
}

X-ray Free-electron lasers (FELs), with the capacity of generating ultrashort, highly coherent and extremely brilliant photon pulses, have given us the first chance to explore the structures and dynamics of the atomic and molecular system at femtosecond time scales and angstrom space scales. To date, several X-ray FEL facilities have been constructed worldwide $[1,2,3,4,5,6]$ and have already enabled the observation and control of very fast phenomena at the atomic time scale, providing an ideal tool in various subjects such as femtochemistry, ultrahigh-resolution imaging, and the investigation of the dynamics in atomic and biological systems $[7,8,9]$. Of particular interest is the experiment performed at seeded X-ray FELs, which have the major advantage of full coherence, precisely arrival time control, uniform longitudinal profile and so on. For most of FEL experiments, a precise knowledge of the characteristics of FEL pulses shot-by-shot is critical and highly desired. Among these pulse properties, the temporal shape of FEL pulses is essential for researches with time resolution much shorter than 
the pulse length. Hence, one of the crucial challenges for FEL experiments is the development of methods and technologies which enable us to measure the temporal profiles of FEL pulses on a shot-to-shot basis.

Methods for extracting the temporal profiles of FEL pulses can be roughly divided into two categories. One is by directly measuring the FEL pulses and the other is based on electron bunch diagnostics.

The most direct approach is to analyze the properties of the radiation pulse itself. The statistical analysis of the FEL spectra $[10,11,12]$ provides a relatively easy way to determine the average temporal width of radiation pulses. But this method is strictly applicable only to non-saturated self-amplified spontaneous emission (SASE) sources since it is based on several assumptions derived from physics of the FEL process, such as the Gaussian distribution for the electron bunch and the Gamma distribution for the radiation energies probability distribution $[13,14]$. A more straightforward determination of the pulse length is possible with autocorrelation [15] and cross-correlation $[16,17]$ techniques. The autocorrelation requires pulse splitting and recombination while the cross-correlation needs two different pulses. Hence, they heavily rely on suitable materials for short wavelength pulses control and interaction. Additionally, the spectral interference pattern generated by two FEL pulses has been acquired to analyse the spectro-temporal characterization by means of the SPIDER algorithm [18]. But this method requires special setup of the machine and thus has limited scope of application. Another direct method, terahertz $(\mathrm{THz})$ field streaking $[19,20]$ has also been applied to reconstruct the temporal shape of FEL pulse. By measuring the momentum change of the photoelectrons which have been streaked in a strong $\mathrm{THz}$ field, single-shot photoelectron spectra can be analyzed and the temporal profile can be reconstructed.

On the other hand, there are indirect methods to acquire the FEL temporal profiles by measuring electron bunches after lasing. One of them is based on the streaking of the electron bunches with a transverse deflecting cavity (TCAV) which, in combination with a dispersive energy spectrometer, can provide a measurement of the electron beam longitudinal phase space[21, 22, 23]. The reconstruction of the temporal profile of a single-photon pulse becomes possible when comparing the phase space distributions between "lasing-on" and "lasing-off" shots. Specifically, the "lasing-off" phase space is chosen from a pre-recorded set which causes mismatches between "lasing-on" and "lasing-off" shots. This always leads to irrational predictions especially when the radiation power is relatively weak. Sometimes even negative power profile occurs due to shot-to-shot fluctuations of the electron beam longitudinal phase space and instability of the RF phase of the deflecting cavity, et cetera.

In this paper, we proposed an online method to retrieve FEL pulses shot-by-shot at the Shanghai Soft X-ray FEL facility (SXFEL). This method designed for seeded FEL is an extension of what was discussed in Ref.[22]. Instead of building a set of "lasing-off" shots, the initial electron beam centroid energy and energy spread at the interaction point between seed laser pulses and electron bunches are evaluated by the Hermite interpolation since the seed laser pulses are much shorter than the electron bunches. This avoids the erroneous measurement inherent to the original algorithm and makes it reliable to characterize the properties of the FEL pulses shot-by-shot during the machine tuning. Based on this method, we have directly observed the electron beam splitting 
process and analyzed the correlation of the FEL properties in real time, which helped us to realize the lasing and parameters optimization of the SXFEL. Our experiments also indicate the feasibility to construct a timing diagnostic and feedback system for the electron beam and an external laser source with a resolution better than $3 \mathrm{fs}$. This has been proved to be very useful to stabilize the seeded FEL output and paves a new way for interpretation of user experiment data in the future.

\section{Results}

Temporal power profile reconstruction of a seeded X-ray FEL. As the first Xray FEL facility in China, the SXFEL is a seeded FEL aiming at generating soft X-ray radiation from a $266 \mathrm{~nm}$ conventional seed laser through a two stages cascaded high-gain harmonic generation (HGHG) configuration $[24,25,26]$. The schematic layout is shown in Fig.1. The first stage is a normal HGHG which consists of two undulator sections separated by a dispersion section. The electron beam first interacts with an external seed laser at $266 \mathrm{~nm}$ to generate sinusoidal energy modulation in the electron beam longitudinal phase space. After a small dispersion chicane (DS), the energy modulation has been converted into electron density modulation (micro-bunching) which contains frequency components at high harmonics of the seed laser. With this kind of electron beam, coherent $44.3 \mathrm{~nm}$ (6th harmonic) radiation pulses are generated and amplified in the following radiator undulator. The output of HGHG inherits the proprieties of the seed laser with high degrees of coherence and stability. However, the harmonic up-conversion number of an HGHG is limited due to the conflict requirements of the electron energy spread between harmonic multiplication and FEL amplification [4, 27, 28]. To cover the X-ray wavelength range, multiple stages of HGHG with the "fresh bunch" (FB) technique are generally required [29, 30, 31]. As the seed laser pulse is much shorter than the electron bunch length, the FEL pulse obtained from the intermediate radiator can be served as the seed laser to interact with the "fresh" part of the electron beam in the following stage and generate higher harmonic radiation pulses at $8.8 \mathrm{~nm}$. At the SXFEL, the pulse duration of seed laser is about $150 \mathrm{fs}$ (FWHM) which is much shorter than the length of electron bunch of about 1.4 ps (FWHM) at the undulator entrance. In order to characterize the FEL pulses, an X-band RF deflector which streaks the electron beam horizontally and a dipole magnet which disperses the beam vertically have been installed downstream of the undulator section. This enables the acquirement of the time-energy longitudinal phase space images of the electron beams on the screen. The detailed experiment set-up can be found in Methods.

Different from FELs that operate with the self-amplified spontaneous emission (SASE) principle [32, 33], cascade seeded FELs usually require electron beams with sufficient longitudinal uniform regions to support the fresh-bunch technique. In addition, the region of the electron bunch to be seeded in modulators is relatively small when comparing to the entire electron bunch. These facts make it reasonable to estimate the initial central energy and energy spread distributions from the longitudinal phase space after lasing. In order to demonstrate this method, start-to-end simulations with all components and parameters (see Methods) of SXFEL have been performed.

The simulated image of the electron beam longitudinal phase space on the screen 


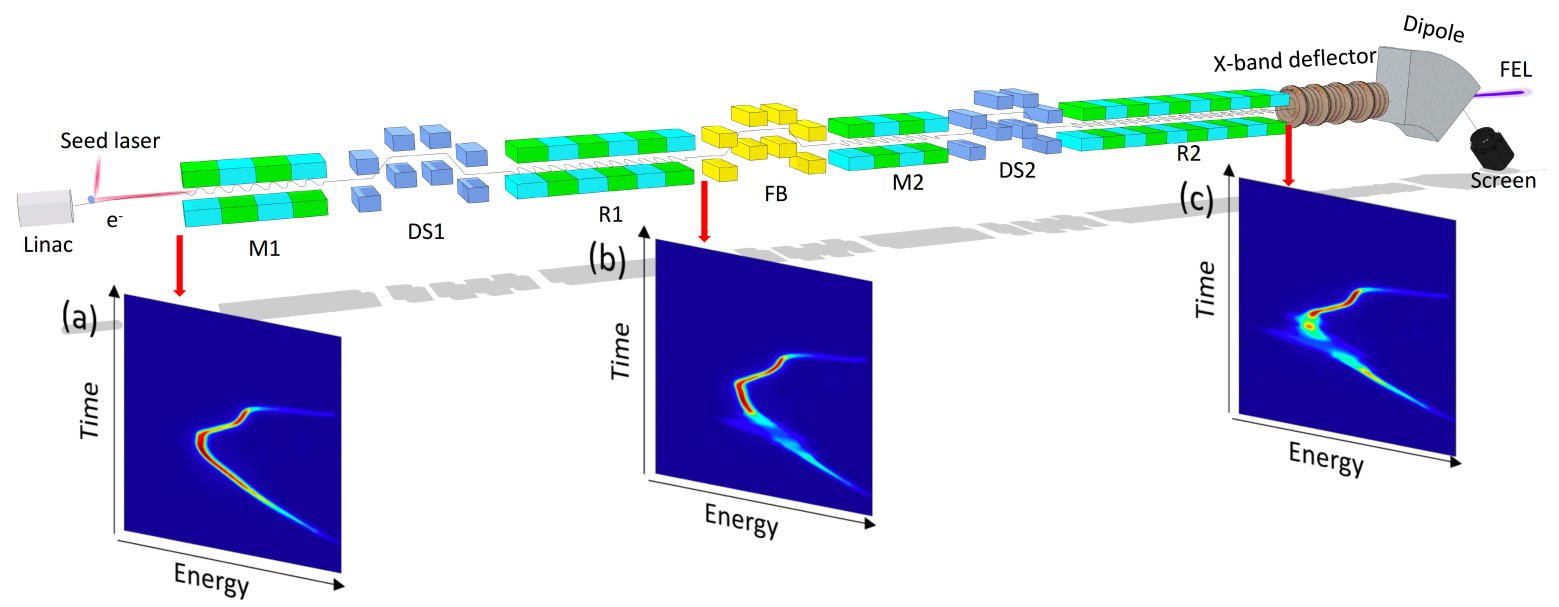

Figure 1: Schematic diagram of SXFEL undulator line. Schematic layout of the two stages cascaded HGHG undulator line at SXFEL. (M:Modulator, DS:Dispersion section, R:Radiator. FB: Fresh-bunch chicane) The panels (a-c) show the measured electron beam longitudinal phase space images on the screen before lasing, after first stage and after second stage, respectively. The bunch charge is about $580 \mathrm{pC}$ with the beam energy of $794 \mathrm{MeV}$. The bunch head is to the top.

after lasing are shown in Fig.2(a) with $x$ corresponding to energy, and $y$ corresponding to time. The induced additional energy spread leaves a conspicuous imprint of FEL radiation from first stage (close to the tail) and second stage (close to the head) on the longitudinal phase space.

By 'slicing' Fig.2(a) along the time dimension, we can obtain the time-dependent electron beam parameters after lasing. The orange and red dash line in Fig.2(a) and black solid line in Fig.2(b) represent the electron beam current profile $I\left(t_{i}\right)$, the central energy after lasing $E_{o n}\left(t_{i}\right)$ and the FEL-induced energy spread in each time slice $t_{i}$, respectively. Instead of many longitudinal spikes in the SASE mode, there are only two main peaks of the energy spread curve for cascaded HGHG mode. One is the result of FEL radiation from the first stage (left) and the other is from the second stage (right). Shouldering the first main peak, there are two small side spikes that are originated from radiation in the magnets of the second stage. This phenomenon has also been observed during the experiments and will be analyzed in the following section. The blue dots in Fig.2(b), referring as the interest points, mark the strongest lasing part of the electron beam in the first and second stages.

In Fig.2(b), the first interest point, corresponding to the peak of the first stage radiation pulse, can be easily found with the continuous wavelet transform-based peak detection algorithm[34]. The fresh-bunch chicane is used to delay the electron beam so that the first-stage FEL pulse can interact with fresh electrons. The delay distance can be accurately calibrated with the set of the fresh bunch chicane. This gives a general position of the second interest point which is then precisely chosen as the maximum point near the estimated position. The red dots in panel (b) are the local minima near the second interest point.

The first main peak of the central energy curve and the region between the red dots in Fig.2(b) define the lasing part of the electron beam in the first and second stages. The distribution of the electron beam central energy without lasing, denoted $E_{\text {off }}\left(t_{i}\right)$, 

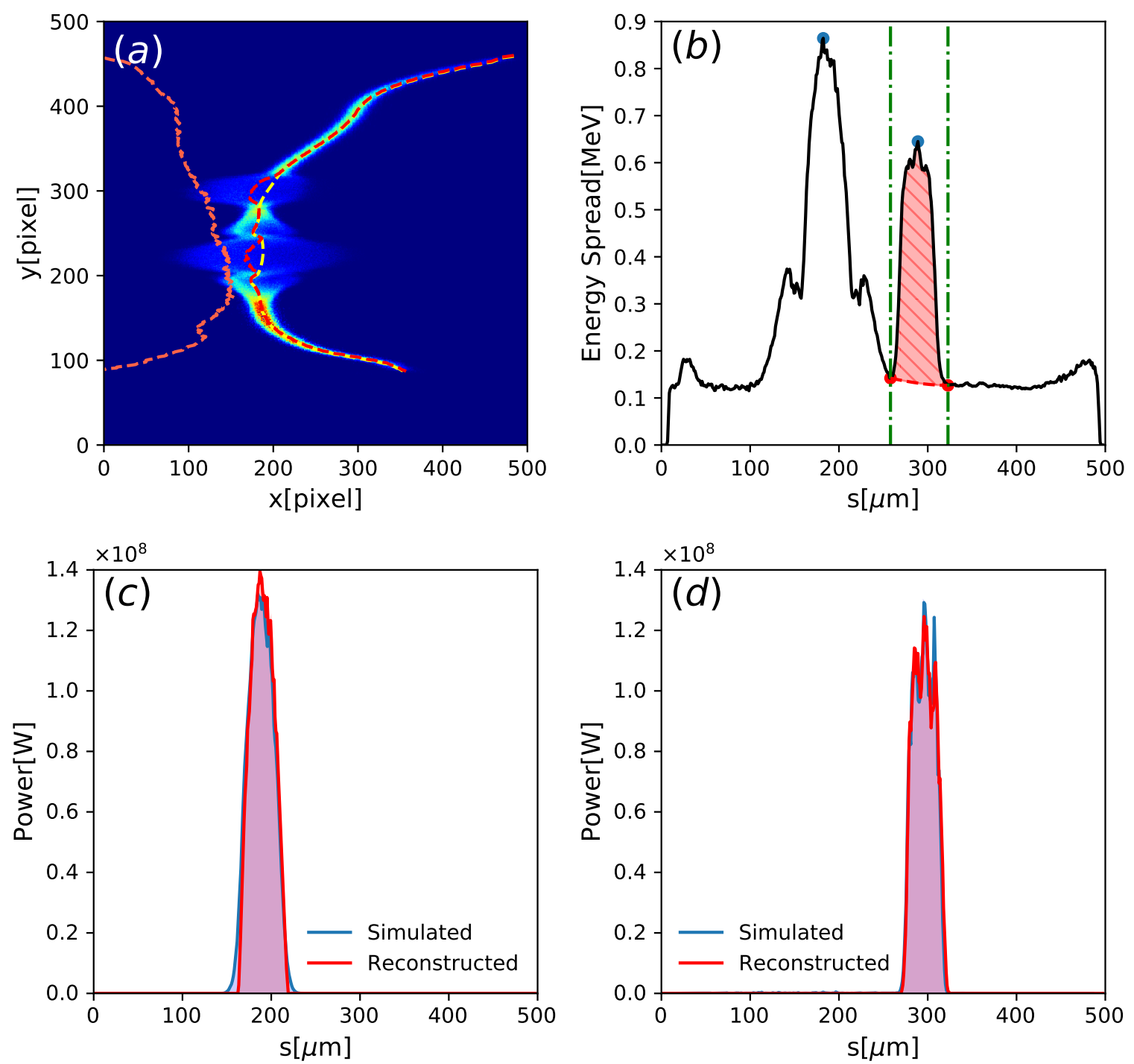

Figure 2: Reconstruction of FEL power profile. (a) The simulated electron beam longitudinal phase space image on the screen after lasing. The current profile and central energy are shown as orange and red dashed line. The yellow dashed line is the estimation of central energy after removing the effects of FEL lasing process. (b) The induced energy spread change due to the FEL amplification. $(\mathbf{c}, \mathbf{d})$ The reconstructed X-ray power profile (red) comparing with the simulated ones (blue) of the first (c) and second (d) stages. The bunch head is to the top in (a) and to the right in (b-d).

can be estimated by the Hermite interpolation represented as the yellow dashed line in Fig.2(a).

With the obtained time-resolved central energy loss, the absolute FEL power profile $P\left(t_{i}\right)$ can be directly determined as $P\left(t_{i}\right)=\left(E_{\text {off }}\left(t_{i}\right)-E_{\text {on }}\left(t_{i}\right)\right) \times I\left(t_{i}\right) / e$ due to the conservation of energy, where $e$ is the elementary charge. Another alternative method to retrieve the FEL power profile is based on the energy spread increase. This can be written as $P\left(t_{i}\right) \propto P_{c a l}\left(t_{i}\right)=I\left(t_{i}\right)^{2 / 3} \times\left(\sigma_{E, \text { on }}^{2}\left(t_{i}\right)-\sigma_{E, o f f}^{2}\left(t_{i}\right)\right)$ (Ref.[35, 36]), where $\sigma_{E}\left(t_{i}\right)$ is the RMS slice energy spread and the indices in $\sigma_{E}$ represent after and before lasing, respectively. The $2 / 3$ power scaling of the current comes from the pierce parameter $\rho$ 
which is proportional to $I^{1 / 3}$. The scale factor is found by normalizing the integral of calculated $P_{c a l}\left(t_{i}\right)$ to the independently measured FEL pulse energy from a calibrated detector. These two methods generally agree very well during the high-gain regime (pre-saturation) of FELs as indicated in Ref.[22]. The approach utilizes the central energy loss are less sensitive than the one based on the change in energy spread, but it can be used to measure the pulse energy and peak power directly. The results in this section and throughout are calculated by the central energy loss.

Figure.2(c,d) represent the reconstructed (red) and simulated (blue) X-ray FEL power profile in the first and second stages, respectively. The pulse lengths and pulse energies for the simulated and reconstructed FEL pulse profile of the first stage are around $116.12 \mathrm{fs}$ (FWHM) and $16.58 \mu \mathrm{J}, 114.98 \mathrm{fs}$ (FWHM) and $16.27 \mu \mathrm{J}$, respectively. Those of the second stage are about $104.69 \mathrm{fs}$ (FWHM) and $13.50 \mu \mathrm{J}$ for the simulation, $105.41 \mathrm{fs}(\mathrm{FWHM})$ and $13.48 \mu \mathrm{J}$ for the reconstruction.

These simulation results demonstrate that the proposed method can be applied to accurately characterize the FEL pulse by single shot. In the following sections we will show how to optimize the seed FEL performance based on this technique.

Optimization of the first stage. In a cascaded HGHG FEL, the radiation generated in the first stage is served as the seed in the second stage. Thus, the intermediate processes between electron beam and seed laser in the first stage play an important role in the determination of the output-pulse characteristics.

For the first stage, the amplitude of bunching factor at $n$th harmonic of the seed and at longitudinal coordinate $\zeta$ is $\left|b_{n}(\zeta)\right|=\exp \left[-0.5\left(k_{n} \sigma_{\gamma} R_{56}\right)^{2}\right] J_{n}\left[k_{n}(\Delta \gamma / \gamma) R_{56}\right]$ (Ref.[39]), where $J_{n}$ is a Bessel function of the first kind of order $n, k_{n}=2 \pi n / \lambda_{0}$ is the wave number of the $n$th harmonic of the seed, $\sigma_{\gamma}$ denotes the electron beam energy spread, $\gamma$ is the electron energy in the units of the rest energy, $R_{56}$ represents the strength of the dispersion section and $\Delta \gamma$ corresponds to the energy modulation induced in the modulator.

At a given harmonic order $n$ and longitudinal amplitude of the seed electric field which is related to $\Delta \gamma$, the strength of dispersion section dominates the bunching factor, which further influences the FEL radiation proprieties. One of the most important and effective approaches to optimize the HGHG FEL (or the first stage of cascaded HGHG FEL) is the tuning of the dispersion strength. A relatively modest change in the settings of the dispersion strength will result in the "pulse splitting" effect [37, 38, 39], which has essential impacts on the output-pulse profiles. The pulse splitting effect has been intensely investigated for seeded FELs, but only indirect measurements with spectrometer have been performed. Here, by using the proposed technique, we can directly observe the evolution of FEL pulse profiles during the beam-splitting process.

The first stage HGHG was optimized for 6 th harmonic generation. The $R_{56}$ of the dispersion chicane was scanned from about $0.10 \mathrm{~mm}$ to $0.24 \mathrm{~mm}$. The longitudinal phase spaces of the electron beams and the corresponding spectra of radiation pulses were recorded by a yttrium aluminium garnet (YAG) screen and a spectrometer simultaneously. The experimental results are illustrated in Fig.3. Starting at $R_{56}=0.17 \mathrm{~mm}$, the pulse splits into two well-separated sub-bunches since the electrons in the center of the laser pulse are overbunched while those close to the head and tail are optimally 

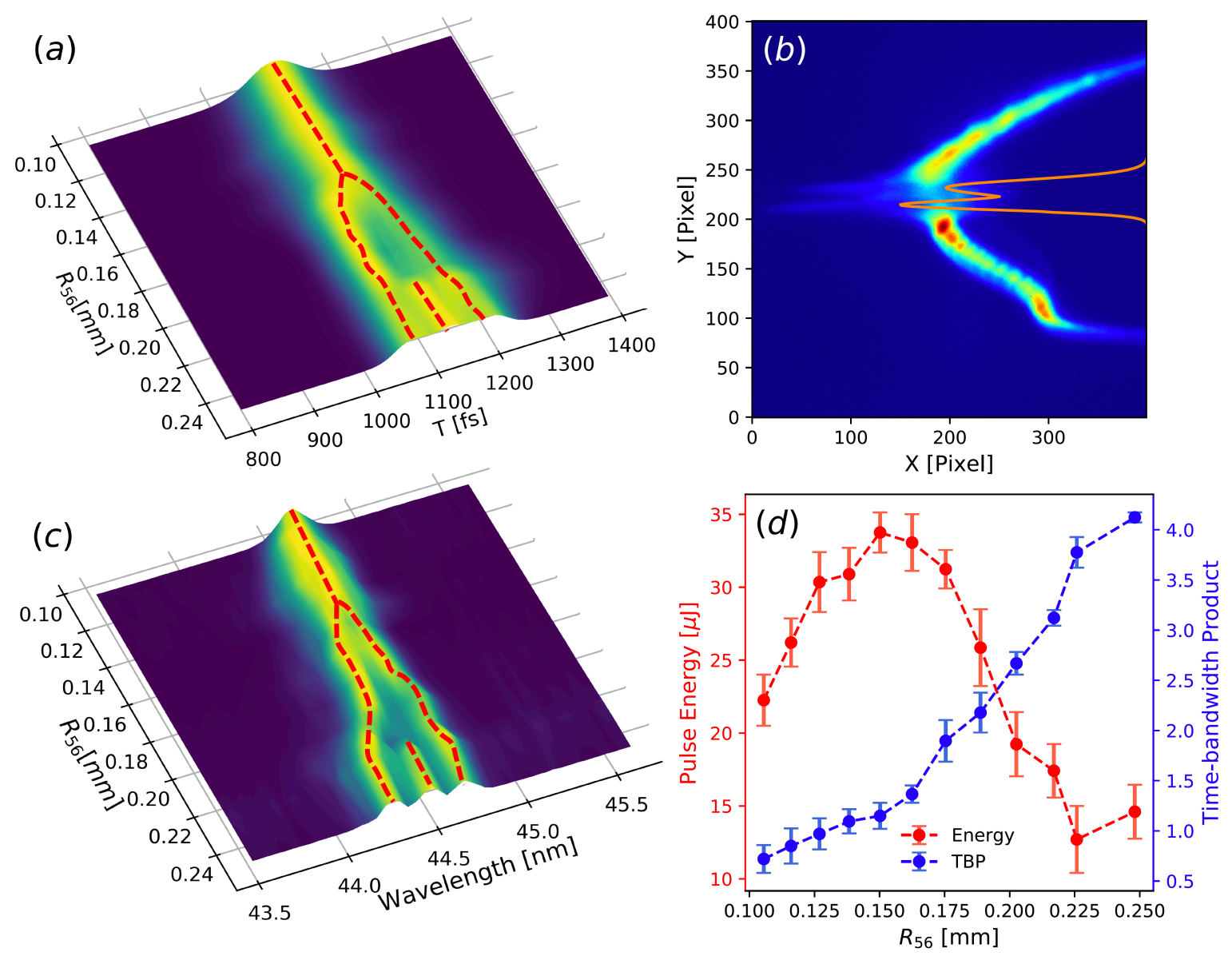

Figure 3: Experimental results for pulse splitting when optimizing the first stage. (a) Pulse longitudinal profiles as a function of $R_{56}$. (b) A typical split phase space and the corresponding FEL power profile (orange) reconstructed with the proposed method. $R_{56}$ equals to $0.20 \mathrm{~mm}$. (c) The splitting phenomena of pulse spectra which are measured simultaneously by the spectrometer. (d) The evolution of FEL pulse energy (red) and the time-bandwidth product (blue) as the $R_{56}$ increases.

bunched as shown in Fig.3(b). This phenomenon is also the source of the two side spikes in Fig.2(c). After lasing in the first stage, this part of electron beam developed very strong microbunching, which was overbunched by the following FB chicane and emitted coherent radiation in the downstream magnets. This process leaves an imprint on the longitudinal phase space and will narrow the suitable lasing region of the electron bunch for the second stage.

As $R_{56}$ gets larger to around $0.22 \mathrm{~mm}$, the pulse splits into three sub-bunches. The recurrence of the central sub-bunch is the result of the Bessel function reaching its second local maximum as $R_{56}$ increases. The simultaneously measured pulse spectra, shown in Fig.3(c), have a similar splitting phenomena as the FEL pulse profiles. By combining these two results, figure 3(d) illustrates the evolution of the FEL pulse energy and the time-bandwidth product (TBP) with the increasing of $R_{56}$. The TBP experiences a sharp increase when the electron bunch splits from one to two and two to three. The initial TBP is about 0.72 , which is already 1.6 times larger than the Fourier limit. This indicates that a moderate frequency chirp exists in the seed laser $[37,38]$ 
which is induced during the $800 \mathrm{~nm}-266 \mathrm{~nm}$ third harmonic generation and the laser transportation. The maximum of the FEL pulse energies takes place at the onset of the pulse splitting. As a result, we need to scarify the pulse energy for better coherence in the first stage. The timing resolution for above measurements is about $3 \mathrm{fs}$, which is limited by the vertical size of the YAG screen. As the deflector of SXFEL is strong enough to stretch the electron beam vertically, we can easily achieve a resolution better than $1 \mathrm{fs}$. However, under this condition, some fragments of the electron beam will be out of the YAG screen.

Two-stage correlation analysis. Due to the lasing principle of the cascaded HGHG FEL, the output-pulse characteristics are significantly influenced by the properties of the electron beam and the first-stage radiation. The correlation analysis between two stages is not only instructive for machine commissioning but also beneficial for understanding the cascaded FEL process.

Longitudinal phase spaces for about 700 consecutive shots have been acquired during the machine tuning. Figure $4(\mathrm{a}, \mathrm{b})$ present two typical measurements of electron beam longitudinal phase spaces after two-stage HGHG. The FEL-induced energy spread changes (yellow dashed line) and the current profiles (orange dashed line) can be obtained through these images. The place of the largest FEL-induced energy spread growth indicates the position of the strongest FEL lasing. In the external seeded FEL, this place is highly related to the seed laser and can serve as a longitudinal position indicator of the seed laser(red solid line). Additionally, the indicator of electron beam longitudinal position(magenta solid line) can be derived from the currents where a specified level is reached (in this experiment we set at $5 \%$ of the currents' maximum value). The distance between these two lines, denoted $\Delta T_{L-E}$, quantifies the relative timing between the external laser and the electron beam. The yellow areas in Fig.4(a,b) are the "golden" regions of electron bunches. Compared with other parts, this "golden" region has much more homogeneous and suitable distributions, such as the sufficient beam current, the good-quality longitudinal and transverse phase space and so on.

With the proposed method, the FEL power profiles for the first stage and second stage can be reconstructed simultaneously. The pulse energies, pulse lengths as well as the relative timings are shown in Fig.4(c-h). The Spearman's correlation coefficients for panel(c-h) are $-0.668,0.440,-0.672,0.707,-0.439$ and -0.594 , respectively. These strong correlations indicate that the relative timing between electron bunches and seed laser pulses plays an essential role in cascaded FEL process. As $\Delta T_{L-E}$ decreases (from Fig.4(a) to Fig.4(b)), a part of electrons in the "golden" region, which are reserved for the second stage, have interacted with the seed laser in the modulator and lased in the radiator of the first stage. Thus, the FEL pulse lengths for the first stage have increased while those for the second stage have decreased. This leads to the same trends of FEL pulse energies for the first and second stages. Consequently, the FEL pulse lengths, as well as pulse energies, from first and second stages are negatively correlated as shown in Fig.4(g,h). These measurement results give an important guidance on tuning of the second stage.

Stabilization of the second stage output The result of two-stage correlation anal- 

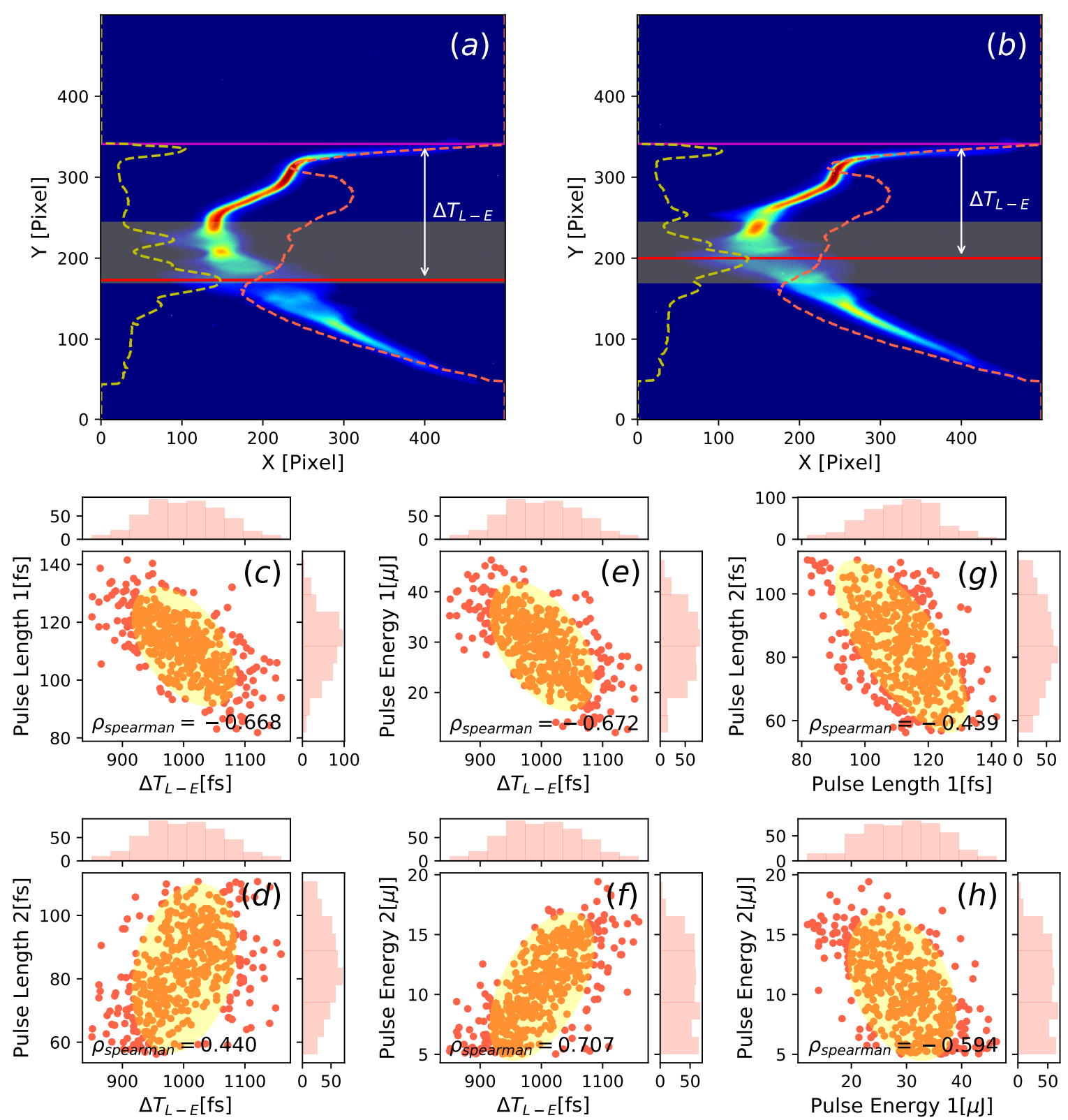

Figure 4: The two-stage correlation analysis. Panel $(\mathbf{a}, \mathbf{b})$ are two typical measured phase spaces with energy spread (yellow) and current (orange) distributions along the electron bunchs. The red and magenta lines mark the longitudinal position of the seed laser and the electron beam, respectively. $\Delta T_{L-E}$ quantifies the relative timing between the seed laser and the electron beam. Panel (c-h) show the correlation between the relatively timings and pulse lengths or pulse energies, and between the first and second stage FEL pulse energies or pulse lengths. The yellow ellipses are the $90 \%$ confidence ellipses.

ysis confirms that the relative timing between the seed laser pulses and the electron bunches needs to be stabilized in order to perform reliable seeding for external seeded FELs. One approach is to install several bunch arrival time monitors (BAMs) which measure the arrival time of an electron bunch relative to an optical reference. This 
optical reference for the monitors can be provided by the all-optical synchronization system[40] based on the mode locked laser pulse train. The most challenging problems are the synchronization of several clock domains and online calibration. Those difficulties make it an extremely complicated system.
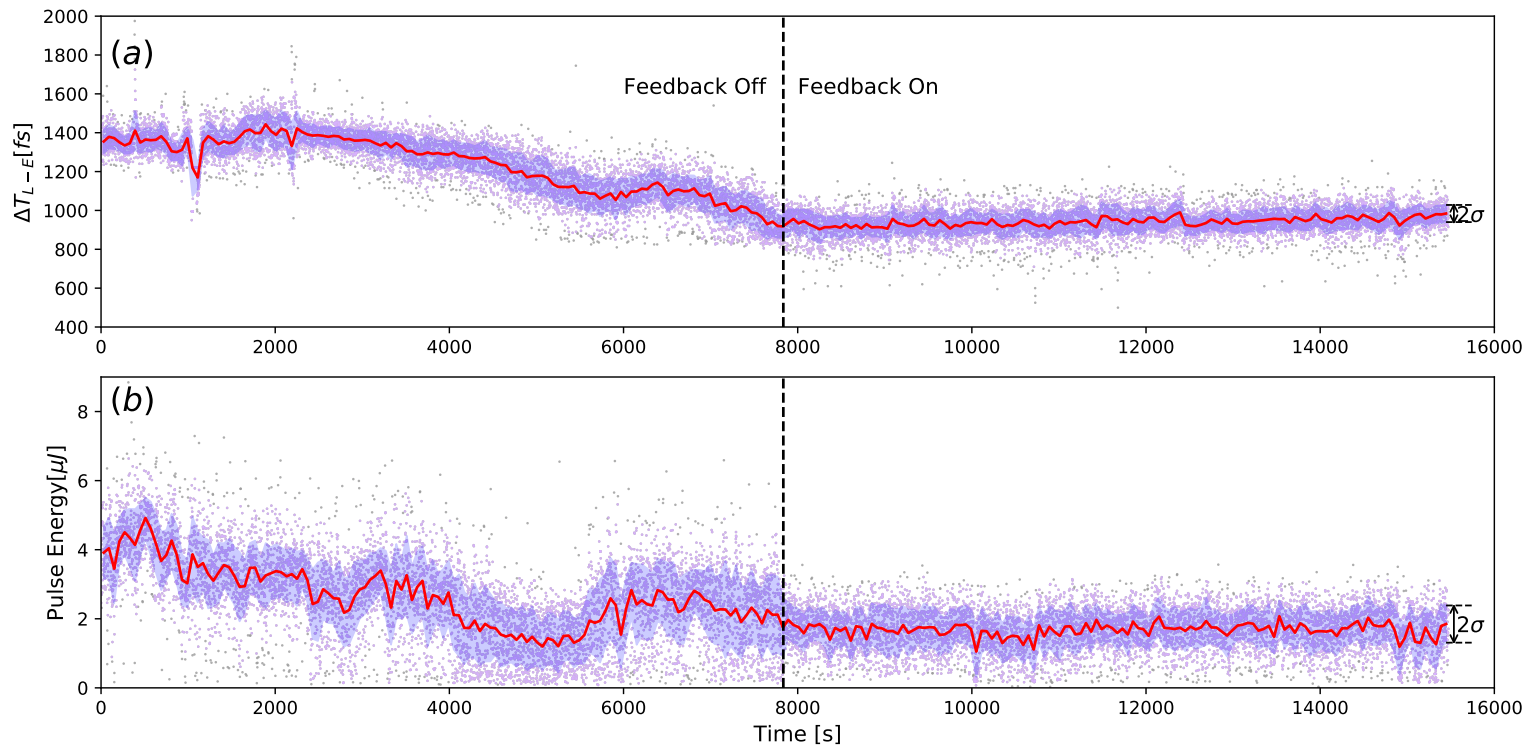

Figure 5: The laser-electron relative timing diagnostics and feedback. A more-than-four-hour continuous acquisition of the laser-electron relative timing (a) and FEL pulse energy in the second stage (b) before and after feedback.

The method proposed above provides a much more straightforward approach to establish a laser-electron relative timing feedback system. $\Delta T_{L-E}$, which is defined in Fig.4(a,b), is served as the objective of the proposed feedback system. Figure.5(a) shows the continuous acquisitions of relative timing between electron bunches and seed laser pulses before and after feedback at SXFEL. More convincingly, the effect of this feedback system on the second-stage FEL pulse energies is shown in Fig.5(b). The amplitudes of drifts have decreased from $525.37 \mathrm{fs}$ to $87.03 \mathrm{fs}$ for relative timings and from $3.82 \mu \mathrm{J}$ to $1.03 \mu \mathrm{J}$ for second-stage FEL pulse energies. The result shows that this method can be used to correct timing drifts between electron bunches and seed laser pulses. It also gives the value of shot-to-shot jitters of the electron beam with respect to an external laser source. The resolution of shot-to-shot timing measurements is about $3 \mathrm{fs}$, which can be further enhanced by increasing the voltage of X-band deflectors and enlarging the YAG screen.

\section{Discussion}

Knowledge of the FEL pulse temporal profiles is of great significance for FEL studies which are aimed at broadening the research frontiers of time-resolved phenomena. The method proposed in this paper can be applied as a robust online single-shot diagnostic tool to measure the X-ray temporal profiles for seeded FELs. Avoiding the explicit choice of baseline from hundreds of "lasing-off" longitudinal phase space images, the 
proposed method provides a reliable and stable stream of X-ray FEL temporal profiles during the FEL tuning process and user experiments. An electron beam and seed laser relative timing feedback system has been established at SXFEL based on the proposed method. The timing drifts between electron bunches and seed laser pulses can be well corrected. It also helps to stabilize the FEL pulse energies of the second stage. This feedback system has become a part of regular operation routines at SXFEL. Additionally, this system also provides the exact values of relative timing between electron beams and external lasers, which may enable researchers to acquire more information in time-resolved experiments.

\section{Methods}

Machine set-up. All experiments are carried out at Shanghai soft X-ray FEL facility (SXFEL). The electron beam is generated by a photocathode RF gun (RF frequency at $2856 \mathrm{MHz}$ ) at a repetition rate of $10 \mathrm{~Hz}$ and can be boosted up to $840 \mathrm{MeV}$ by a linac which consists of $4 \mathrm{~S}$-band and $6 \mathrm{C}$-band linac structures. The electron bunch is compressed to $500 \mathrm{~A}$ by a magnetic chicane. The electron bunch charge is about $580 \mathrm{pC}$ and the electron bunch length at the end of linac is $1.4 \mathrm{ps}$ (FWHM). The relative energy spread is about $10^{-4}$ and the normalized transverse emittance is $1 \mathrm{~mm} \cdot \mathrm{mrad}$. A laser heater has been installed at the beginning of linac to suppress the microbunching instability.

The seed laser is originated from a commercial Ti:Sapphire laser system, which can provide lasers up to $3 \mathrm{~mJ}$ at $800 \mathrm{~nm}$. A third harmonic generation is employed to convert the laser to $266 \mathrm{~nm}(\sim 100 \mathrm{fs})$. The seed laser pulses are elongated to $\sim 150 \mathrm{fs}$ during the propagation.

The SXFEL undulator system consists of two stages of seeded FELs. The first stage contains a permanent magnetic modulator with the period length of $80 \mathrm{~mm}$ and three variable gap radiators which have a period of $40 \mathrm{~mm}$. The momentum compaction $R_{56}$ of the fresh-bunch chicane can be continually tuned from $0 \mathrm{~mm}$ to $10.6 \mathrm{~mm}$ which can shift the first-stage radiation ahead up to $17.7 \mathrm{ps}$. The second stage is composed of a $1.65 \mathrm{~m}$-long modulator (30 periods with a period length of $55 \mathrm{~mm}$ ) and six variable gap radiators. Each radiator is about $3 \mathrm{~m}$ long with a period length of $23.5 \mathrm{~mm}$.

Diagnostics. The longitudinal phase spaces of electron beams are measured by the combination of deflecting cavities and an energy spectrometer. Two 1-meter-long Xband $(11.424 \mathrm{GHz}) \mathrm{rf}$ deflecting structures powered by a $50 \mathrm{MW}$ klystron are adopted to provide a horizontal kick to the electron beam. After a 22-meter-long drift section, a 2meter-long dipole is adopted to vertically disperse the electrons with different energies. Finally, the electron beam is captured by the YAG crystal screen which is installed downstream of the dipole magnet. The temporal resolution is better than $3 \mathrm{fs}$ and the energy resolution is about $36 \mathrm{keV}$.

The spectra of the first stage FEL pulses are measured by a dedicated VUV spectrometer (McPherson, Model 234/302) with the spectral coverage from $40 \mathrm{~nm}$ to $200 \mathrm{~nm}$ and the resolution of $0.05 \mathrm{~nm}$. The FEL pulses from the first stage are reflected by a movable gold mirror placed in the middle of the FB chicane. The longitudinal phase 
space of the electron beam and the spectrum of the FEL pulse can be measured simultaneously.

\section{References}

[1] Ackermann, W. et al. Operation of a free-electron laser from the extreme ultraviolet to the water window. Nat. Photon. 1, 336-342 (2007).

[2] Emma, P. et al. First lasing and operation of an ångstrom-wavelength free-electron laser. Nat. Photon. 4, 641-647 (2010).

[3] Ishikawa, T. et al. A compact X-ray free-electron laser emitting in the sub-ångström region. Nat. Photon. 6, 540-544 (2012).

[4] Allaria, E. et al. Highly coherent and stable pulses from the FERMI seeded freeelectron laser in the extreme ultraviolet. Nat. Photon. 6, 699-704 (2012).

[5] Milne, C.J. et al. SwissFEL: The Swiss X-ray Free Electron Laser. Appl. Sci. 7, 720 (2017).

[6] Kang, H. et al. Hard X-ray free-electron laser with femtosecond-scale timing jitter. Nat. Photon. 11, 708-713 (2017).

[7] Öström, H. et al. Probing the transition state region in catalytic CO oxidation on Ru. Science 347, 978-982 (2015).

[8] Gerber, S. et al. Three-dimensional charge density wave order in $\mathrm{YBa}_{2} \mathrm{Cu}_{3} \mathrm{O}_{6} .67$ at high magnetic fields. Science 350, 949-952 (2015).

[9] Rudenko, A. et al. Femtosecond response of polyatomic molecules to ultra-intense hard X-rays. Nature 546, 129-132 (2017).

[10] Lutman, A. A. et al. Femtosecond x-ray free electron laser pulse duration measurement from spectral correlation function. Phys. Rev. ST Accel. Beams 15, 030705 (2012).

[11] Engel, R., Düsterer, S., Brenner, G. \& Teubner, U. Quasi-real-time photon pulse duration measurement by analysis of FEL radiation spectra. J. Synchrotron Radiat. 23, 118-122 (2016).

[12] Inubushi, Y. et al. Determination of the pulse duration of an x-Ray free electron laser using highly resolved single-shot spectra. Phys. Rev. Lett. 109, 144801 (2012).

[13] Saldin, E.L., Schneidmiller, E.A. \& Yurkov, M.V. Statistical properties of radiation from VUV and X-ray free electron laser. Opt. Commun. 148, 383-403 (1998).

[14] Behrens, C. et al. Constraints on photon pulse duration from longitudinal electron beam diagnostics at a soft x-ray free-electron laser. Phys. Rev. ST Accel. Beams 15, 030707 (2012). 
[15] Mitzner, R. et al. Direct autocorrelation of soft-x-ray free-electron-laser pulses by time-resolved two-photon double ionization of He. Phys. Rev. A. 80, 025402 (2009).

[16] Riedel, R. et al. Single-shot pulse duration monitor for extreme ultraviolet and X-ray free-electron lasers. Nat. Commun. 4, 1731 (2013).

[17] Ding, Y. et al. Femtosecond X-Ray pulse characterization in free-electron lasers using a cross-correlation technique. Phys. Rev. Lett. 109, 254802 (2012).

[18] De Ninno, G. et al. Single-shot spectro-temporal characterization of XUV pulses from a seeded free-electron laser. Nat. Commun. 6, 8075 (2015).

[19] Frühling, U. et al. Single-shot terahertz-field-driven X-ray streak camera. Nat. Photon. 3, 523-528 (2009).

[20] Grguraš, I. et al. Ultrafast X-ray pulse characterization at free-electron lasers. Nat. Photon. 6, 852-857 (2012).

[21] Ding, Y. et al. Femtosecond x-ray pulse temporal characterization in free-electron lasers using a transverse deflector. Phys. Rev. ST Accel. Beams 14, 120701 (2011).

[22] Behrens, C. et al. Few-femtosecond time-resolved measurements of X-ray freeelectron lasers. Nat. Commun. 5, 3762 (2014).

[23] Plath, T. et al. Mapping few-femtosecond slices of ultra-relativistic electron bunches. Sci. Rep. 7, 2431 (2017).

[24] Yu, L. H. et al. High-gain harmonic-generation free-electron laser. Science 289, 932-934 (2000).

[25] Yu, L. H. et al. First Ultraviolet High-Gain Harmonic-Generation Free-Electron Laser. Phys. Rev. Lett. 91, 074801 (2003).

[26] Wu, J. \& Yu, L. H. Coherent hard X-ray production by cascading stages of High Gain Harmonic Generation. Nucl. Instrum. Methods A 475, 104-111 (2001).

[27] McNeil, B. W. J., Robb, G. R. M., Poole, M. W. \& Thompson, N. R. Harmonic Lasing in a Free-Electron-Laser Amplifier. Phys. Rev. Lett. 96, 084801 (2006).

[28] Schneidmiller, E.A. \& Yurkov, M.V. Harmonic lasing in x-ray free electron lasers. Phys. Rev. ST Accel. Beams 15, 080702 (2012).

[29] Yu, L. H. \& Ben-Zvi, I. High-gain harmonic generation of soft x-rays with the "fresh bunch" technique. Nucl. Instrum. Methods A 393, 96-99 (1997).

[30] Liu, B. et al. Demonstration of a widely-tunable and fully-coherent high-gain harmonic-generation free-electron laser. Phys. Rev. ST Accel. Beams 16, 020704 (2013). 
[31] Allaria, E. et al. Two-stage seeded soft-X-ray free-electron laser. Nat. Photon. 7, 913-918 (2013).

[32] Kondratenko, A. M. \& Saldin, E. L. Generating of coherent radiation by a relativistic electron beam in an ondulator. Part. Accel. 10, 207-216 (1980).

[33] Bonifacio, R., Pellegrini, C. \& Narducci, L.M. Collective instabilities and high-gain regime in a free electron laser. Opt. Commun. 50, 373-378 (1984).

[34] Du, P., Kibbe, W. A. \& Lin, S. M. Improved peak detection in mass spectrum by incorporating continuous wavelet transform-based pattern. Bioinformatics 22, 2059-2065 (2006).

[35] Huang, Z. et al. Steady-state analysis of short-wavelength, high-gain FELs in a large storage ring. Nucl. Instrum. Methods A 593, 120-124 (2008).

[36] Maxwell, T. J. et al. in Proceedings of SPIE (Femtosecond-scale x-ray FEL diagnostics with the LCLS X-band transverse deflector) Vol. 9210, 92100J (SPIE, United States, 2014).

[37] Labat, M. et al. Pulse Splitting in Short Wavelength Seeded Free Electron Lasers. Phys. Rev. Lett. 103, 264801 (2009).

[38] Gauthier, D. et al. Spectrotemporal Shaping of Seeded Free-Electron Laser Pulses. Phys. Rev. Lett. 115, 114801 (2015).

[39] Finetti, P. et al. Pulse Duration of Seeded Free-Electron Lasers. Phys. Rev. X 7, 021043 (2017).

[40] Schulz, S. et al. Femtosecond all-optical synchronization of an X-ray free-electron laser. Nat. Commun. 6, 5938 (2015).

\section{Acknowledgments}

The authors would like to thank Haixiao Deng, Tao Liu, Zhen Wang, Dao Xiang, Shanchuan Yan for useful comments on the experiments and simulations. Many aspects of this work have benefited a lot from the discussion with Jiawei Yan and Xiaofan Wang. This work is supported by the National Key Research and Development Program of China (No. 2016YFA0401901), National Natural Science Foundation of China (11975300) and Shanghai Science and Technology Committee Rising-Star Program (20QA1410100).

\section{Author Contributions}

B.L. and Z.T.Z guided the work and organized the experimental activities. L.Z., D.G. and B.L. develop the code used in the experiment. L.Z., C.F., D.G. and B.L. conducted the experiment on the accelerator and FEL at SXFEL. L.Z. and C.F. carried out numerical simulations and analysed the experiment data. L.Z. and C.F. wrote the manuscript draft. All authors discussed and contributed to improving the final version of the manuscript.

\section{Competing Interests statement}

The authors declare no competing interests. 